\title{
Comparison of Heart Rhythm and Morphological ECG Features in Recognition of Sleep Apnea from the ECG
}

\author{
C Maier ${ }^{1}$, H Dickhaus ${ }^{1}$, M Bauch $^{1}$, T Penzel $^{2}$ \\ ${ }^{1}$ University of Applied Sciences Heilbronn, Heilbronn, Germany \\ ${ }^{2}$ Department of Internal Medicine, Philipps-University, Marburg, Germany
}

\begin{abstract}
This study addresses the problem of sleep apnea recognition on a minute-by-minute basis from single-lead ECGs recorded overnight. Analysis of heart rate fluctuations, quantified by the series of RR-intervals, is compared to analysis of ECG morphology variations, assessed using signal vectors from the QRS- and the T-wave region and projecting them onto their first principal component. The resulting series of scalar Karhunen-Loève coefficients (KLCs) were used as descriptors of morphology. From the derived series, we calculated a measure of similarity and a spectral index in temporal segments of 5 min, and assessed their diagnostic accuracy by ROCanalysis. Although the performance for the RR-series and the similarity feature was $81 \%$ / $84 \%$ sens ./ spec., better results up to $87 \%$ / $87 \%$ were obtained from the T-wave KLCs. It is concluded that the effects of sleep apnea on the ECG are reflected more uniformly in morphology variations of the ECG compared to heart rhythm.
\end{abstract}

\section{Introduction}

The prevalence of the obstructive sleep apnea syndrome (OSAS) is estimated to be $4 \%$ in middle-aged men and $2 \%$ in women [1]. Patients are exposed to an increased risk of mortality, mainly due to cardiovascular events. The survival rate significantly decreases with higher degree of severity of OSAS. Beside detailed analysis of medical history, the gold standard in diagnosis of OSAS is polysomnography, a costly procedure comprising multi-channel recordings of respiratory flow, noise, and movements as well as saturation of peripheral oxygen, heart rate, EEG, EOG, and EMG, which is only practicable in specially equipped sleeping laboratories. Due to the high prevalence of OSAS, paired with increased incidence of cardiovascular complications, simpler and less costly methods for diagnosis and estimation of severity are highly desirable and intensively searched for. Under the condition of a reliable recognition rate, its practicability and little cost suggest analysis of the ECG as a favourable tool for this purpose. Guilleminault et al. [2] have described a characteristic pattern of heart rate change - cyclic variation of heart rate (CVHR) - mediated by the autonomous nervous system (ANS) during ongoing phases of apnea. Moody et al. [3] suggest a method to assess respiratory activity from amplitude variations of the ECG, and use this information for apnea detection.

Recently, within the scope of the CinC Challenge 2000, quantification of both phenomena has been applied successfully to the identification of time (minutes) spent in phases of apnea. Best results were generally obtained from spectral measures of heart rate variability and ECG amplitude variations [4], however a comparison of the relative merits appears difficult since the participating groups have used mixed approaches, different strategies for normalization and classification, and the dimension of the feature space often was rather high.

In this study, we also address the problem of sleep apnea recognition from single-lead ECGs recorded during night-time. To assess whether the effects of sleep apnea on the ECG are more clearly manifested in heart rhythm fluctuations or ECG morphology variations, we analyze both sources of information separately using the same set of features, and give a comparison of the results.

\section{Material and methods}

The data we used in this study was taken from the annotated learning set of the Physionet [5] Apneadatabase. It consists of 35 single-channel overnight ECGrecordings, each $\sim 8 \mathrm{~h}$ in duration, with a sampling rate of $100 \mathrm{~Hz}$. For each record, minute by minute annotations on the presence or absence of apnea episodes are available. Due to partly severe noise contaminations, we excluded signal b05 and some corrupted segments in the remaining signals from analysis. The total duration of analyzed signals was 16317 minutes.

After upsampling the ECG signals to $1000 \mathrm{~Hz}$ by means of cubic spline interpolation, QRS-complexes were detected and classified as either normal beats or ectopics. Special care was taken on consistent fiducialtriggers. From the QRS occurrences, we calculated the interbeat-interval (RR) series as well as series of Karhunen-Loève (KL) projection-coefficients on the first principal component. The latter were derived from two 


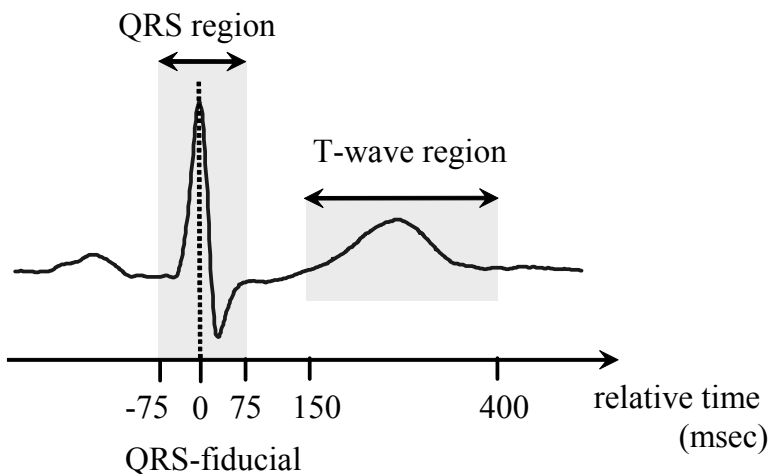

Fig. 1. Regions used for calculation of the KarhunenLoeve basis vectors. Time is relative to the $\mathrm{R}$-wave fiducial point.

different phases of the ECG cycle located relatively to the QRS fiducial point (Fig. 1). Before, we performed a baseline correction by subtracting a median filtered version of the ECG (width $125 \mathrm{~ms}$ ) in the QRS-region. For the T-wave, we smoothed the signal using a $2^{\text {nd }}$ order Savitzky-Golay filter of width $125 \mathrm{~ms}$ and then removed the linear trend fitted to the ECG from $\mathrm{R}+100 \mathrm{~ms}$ to $\mathrm{R}+450 \mathrm{~ms}$.

For each ECG cycle $\mathrm{n}$, vectors $\vec{x}^{\text {qrs }}(n)$ and $\vec{x}^{t}(n)$ comprising the QRS region ( $\mathrm{R}-75 \mathrm{~ms}$ to $\mathrm{R}+75 \mathrm{~ms}$ ) and the $\mathrm{T}$-wave region $(\mathrm{R}+150 \mathrm{~ms}$ to $\mathrm{R}+400 \mathrm{~ms})$ were extracted. From the ensemble of these vectors, we calculated the respective covariance-matrices according to

$$
C^{q r s}=E\left\{\left(\vec{x}^{q r s}(n)-\vec{m}^{q r s}\right) \cdot\left(\vec{x}^{q r s}(n)-\vec{m}^{q r s}\right)^{\prime}\right\}
$$

where $\vec{m}^{\text {qrs }}$ denotes the mean QRS vector over all ECG cycles n, and ' the transposed vector.

$$
\vec{m}^{q r s}=\frac{1}{N} \sum_{n=0}^{N-1} \vec{x}^{q r s}(n)
$$

$\vec{\phi}^{\text {qrs }}$ is calculated from $C^{q r s}$ as the eigenvector with the largest eigenvalue. For each ECG cycle n, the projection of the respective QRS-vector $\vec{x}^{q r s}(n)$ on $\vec{\phi}^{\text {qrs }}$ is calculated resulting in one scalar value or $\mathrm{KL}$ coefficient $\alpha^{q r s}(n)$ per heart beat.

$$
\alpha^{q r s}(n)=\left\langle\left(\vec{x}^{q r s}(n)-\vec{m}^{q r s}(n)\right) \cdot \vec{\phi}^{q r s}\right\rangle
$$

Thus, $\alpha^{q r s}(n)$ represents a weighted deviation of beat $\mathrm{n}$ from the mean QRS complex. For the T-wave region, KL-coefficients $\alpha^{t}(n)$ are calculated in an analogous

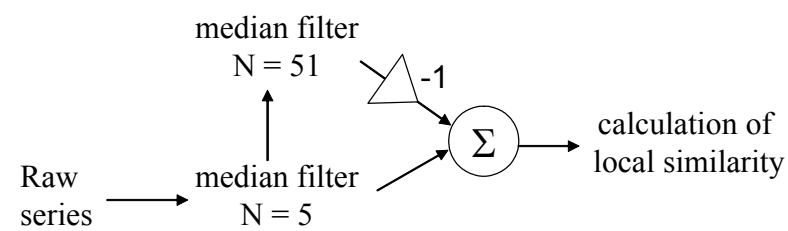

Fig. 2. Nonlinear band-pass filter applied before calculation of local similarity index fashion. RR-intervals and KL-coefficients from ectopic beats or in shorter data gaps were interpolated using a local phase-space prediction algorithm [6].

Both, the RR-interval and the KL-coefficient series served as basis for further feature extraction. Whereas the RR-series contains heart rhythm information, the dynamic evolution of the KL-coefficients reflects changes of amplitude or morphology in the respective phases of the ECG-cycle.

KL-coefficients have been found to successfully reflect ischemic changes in the ST-segment [7]. However, it must be noted that the KL series are generally dependent on posture; changes in posture introduce step-like structures in the time course that we removed using a 201 point median highpass-filter. Features were calculated from temporal segments of 5 min duration that were shifted in time in increments of 1 min over the total record. Since also the absolute variability of the KL-coefficients is likely to be posture dependent, we only used features that were normalized within one segment. This brings the additional benefit that outliers and artefacts in one segment do not corrupt the values of other segments what might happen when within-record standardizations for example to zero mean and unit variance are used.

To capture the locally mostly rather regular CVHRpattern, we defined a measure of local signal similarity (1Simil) based on correlation analysis [8]: Each $5 \mathrm{~min}$ segment was band-pass filtered using a low-pass median filter of width 5, and a high-pass median filter of width 51, emphasizing the CVHR-related pattern (Fig. 2).

From the band-pass series, we extracted the central segment of 1 min duration and shifted it over the total 5 min segment, calculating the normalized correlation coefficient for each time shift (Fig. 3). Since this measure is independent of absolute variability, it can be compared between and within records without any difficulties.
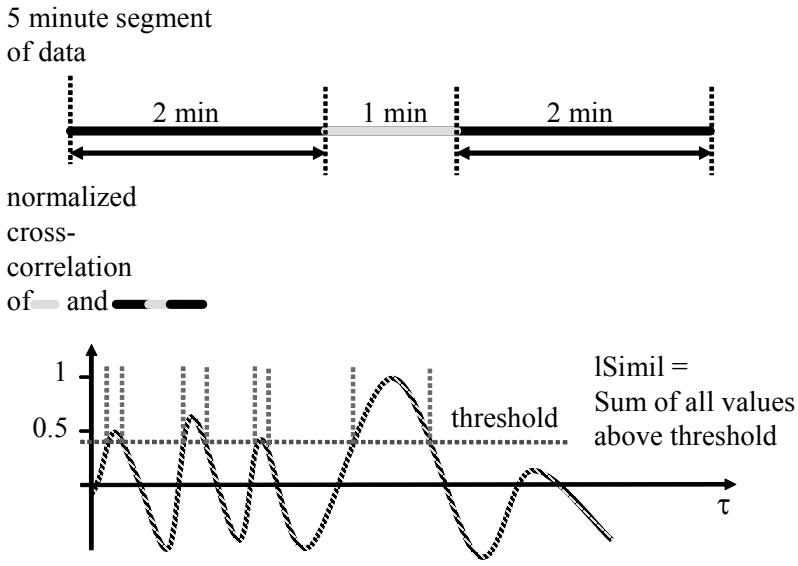

Fig. 3. Calculation of local similarity index 1Simil. The central minute of each 5-min segment is cross-correlated with the whole segment. All values exceeding a threshold are summed. 


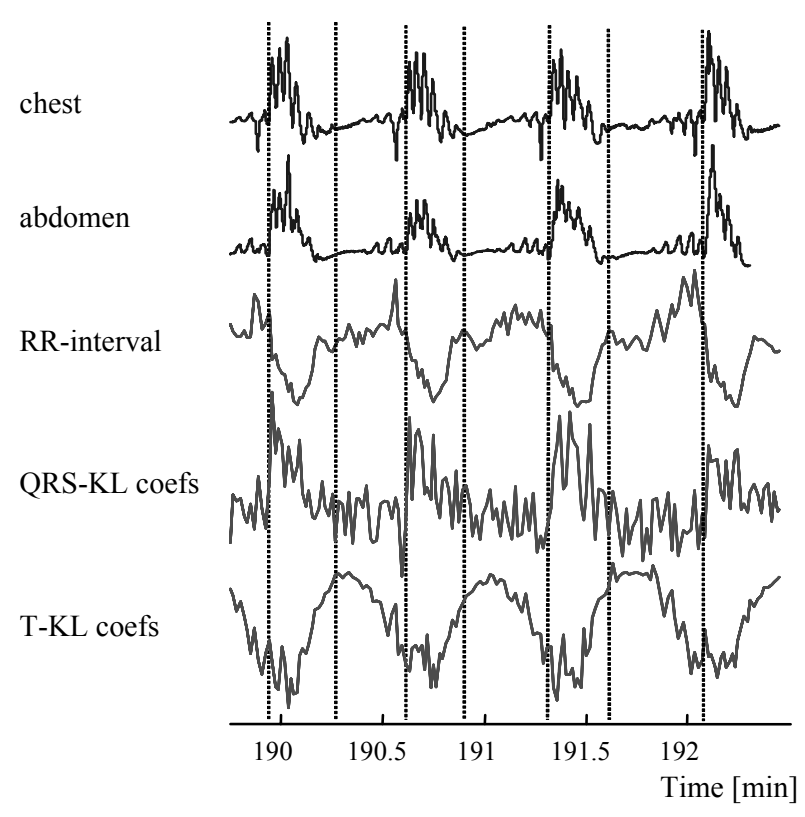

Fig. 4. 2.5 minute time course of chest and abdominal movements (upper traces) and calculated series of RR-intervals ( $3^{\text {rd }}$ trace), QRS KL-coefficients $\left(4^{\text {th }}\right.$ trace) and T-wave KLcoefficients $\left(5^{\text {th }}\right.$ trace) during a phase of apnea. Excerpt of record a04.

For analysis in the frequency-domain, each 5-minute segment of the series was submitted to power spectral density estimation based on the FFT using the Matlab PSD-command. Before, the series were equidistantly resampled at $3 \mathrm{~Hz}$, and the mean was removed. We used a 256 point FFT with a Hanning window and an overlap of 128 points. The frequency resolution of the spectra was $0.012 \mathrm{~Hz}$. To allow for inter-record-comparison and compensate for scaling differences related to changes in posture, we normalized the power of each spectral component to the total power of the 5-min segment. Only the second harmonic, centred at $0.024 \mathrm{~Hz}$ (period 41.7s) was used as marker of activity in the CVHR frequency range. We refer to this component as relPSD_2 $\Delta \mathrm{f}$.

It is important to note that prior to performance evaluation, the time-course of all feature values was smoothed applying a median-filter of width 7. Feature performance was assessed as sensitivity (sens) and specificity (spec) by means of receiver operating characteristics (ROC).

\section{Results}

Visual inspection of annotated plots showed a more or less pronounced CVHR pattern in the RR-series for most of the analyzed records during phases of apnea (Fig. 4, trace 3). Here, the fundamental frequency in the RRseries clearly is the repetition rate of the apnea episodes. However, we observed considerable variability of this pattern between different records and partly even within the same record. In Fig. 4 trace 5, the series of T-KL coefficients demonstrates the same fundamental frequency in an almost sinusoidal form. Moreover, higher frequency-components, obviously related to the strongest respiratory movements, are superimposed. The series of QRS-KL coefficients often contained higher-frequency components, even in the absence of respiratory movements (Fig. 4). Nevertheless, we often observed swings that in position and duration were linked to the CVHRswings seen in the RR-series.

The results of the ROC-Analysis are given in table 1 . For both features, best results are obtained from the series of T-KL coefficients showing a performance of $83.6 \%$ / $85.8 \%$ sensitivity / specificity for relPSD_2 $\Delta$ f and $87.7 \%$ / $87.0 \%$ for 1Simil. All series achieve better class separation with the local similarity feature compared to the spectral coefficient. The strongest increase in performance (about $+6 \%$ sensitivity and specificity to $81.1 \% / 84.5 \%$ ) is found in the RR-series.

\section{Discussion}

Our results show that significant information on sleep apnea is contained in both, heart rhythm and ECG morphology. We were surprised to note that the effects of apnea were reflected even more pronounced in the dynamics of the T-KL-coefficients compared to heart rhythm. However, the results for the spectral component confirm our observation from the annotated plots that the T-KLcoefficients often reflect the frequency of repetition of apnea episodes more uniformly and regularly than the CVHR-swings in the RR-interval series. 83.6\%/85.5\% sensitivity and specificity compared to $75.6 \% / 78.2 \%$ for the RR-series clearly indicate this (table 1).

A possible explanation is that heart rate variability is affected by many additional factors - i.e. different sleep stages - that may overlay and blur the CVHR pattern and reduce the relative power of the CVHR component. Moreover, the flanks of CVHR swings can demonstrate quite sharp accelerations and decelerations of heart rate that contribute to several frequency components whereas in the T-KL series we often observed a sinusoidal or triangular-like shape with smoother transitions.

The improvement of the results for the RR-series when using the local similarity feature supports this interpretation; there is a considerable increase of $6 \%$ in

Table 1. Classification performance of single features derived from RR-series and QRS-/T- KL coefficients

\begin{tabular}{clll}
\hline Feature & Signal & Sens. & Spec. \\
\hline \multirow{2}{*}{ relPSD_2 $\mathrm{ff}$} & T-KL & 83.6 & 85.5 \\
$(0.024-\mathrm{Hz})$ & QRS-KL & 72.7 & 76.7 \\
& RR & 75.6 & 78.2 \\
\hline \multirow{2}{*}{ local } & T -KL & 87.7 & 87.0 \\
similarity & QRS-KL & 74.8 & 78.7 \\
& RR & 81.1 & 84.5 \\
\hline
\end{tabular}


sensitivity and specificity. This feature is not dependent on predefined frequency bands but quantifies the repetition of the most dominant structures in the time domain. Moreover, it is more tolerant against irregularities in the repetition rate of apnea episodes. Probably, the latter is the reason for the further improvement on the T-KL coefficients to $87.7 \% / 87.0 \%$ (Table 1). The fact that a single measure achieves this performance inter-individually using a simple threshold decision is remarkable.

It is well known, that the duration of the RT-interval is modulated by the heart rate, and this modulation is likely to be reflected also in the T-KL coefficients. Insofar, it is not surprising to find a modulation in the CVHR frequency range in this series, too. Nevertheless, it seems surprising that this lower frequency component appears even more pronounced than the higher frequency modulation related to respiratory movements, and visual analysis often revealed marked changes in T-wave morphology that seem hardly attributable solely to variations in heart rate. Moreover, it is not evident why the classification rate obtained from the T-KLcoefficients then is better than the performance of the putatively 'original' RR-series.

From this point of view, the question arises whether additional mechanisms might induce dynamic changes in the T-Wave region during episodes of apnea, what from physiology is plausible since the T-wave, especially the ST-segment, is known to be sensitive to ischaemic events that in more moderate form are conceivable for apnea, too. This also could explain the clearly decreased performance of the QRS-KL coefficients compared to the T-KL-coefficients (table 1), however at this stage we cannot rule out that the observed difference, at least in part, is the result of an artifact due to the low original sampling rate of only $100 \mathrm{~Hz}$. This might bring a loss of signal amplitude in the fast signal changes of the QRS complex that also affects the KL-coefficients. However, this effect should be reduced if the signal has been properly band limited before analog-to-digital conversion. Although we observed a 'high frequency contamination' in the series of QRS-coefficients, even in absence of respiratory movements, there were also many 'clean' QRS-KL series. The properties were generally much more inconsistent compared to T-KL, and could change dramatically even within the same record, presumably related to changes in posture. Multi-channelECGs recorded with a higher sampling rate should be used to resolve whether this is an artifact, a postural effect or a true difference.

\section{Conclusion}

From our results, we can conclude that rhythm as well as morphology features contain important information on the problem of apnea detection. Both phenomena reflect the effects of irregular respiratory activity found during phases of apnea. However, our results suggest that the variability of ECG morphology reflects apnea related patterns more uniformly compared to heart rate. For further development, and to resolve the nature of the difference between properties of the T-Wave and the QRS-complex morphology indices, multi-channel recordings with higher sampling rate should be acquired.

\section{References}

[1] Young T, Palta M, Dempsey J, Skatrud J, Weber S, Badr C. The occurrence of sleep-disordered breathing among middle-aged adults. N Engl. J Med 1993;328: 1230-5.

[2] Guilleminault C, Tilkian A, Dement WC. Cyclical variation of the heart rate in sleep apnoea syndrome. Mechanisms and usefulness of $24 \mathrm{hr}$ electrocardiography as a screening technique. Lancet 1984:1;126-31.

[3] Moody GB, Mark RG, Zoccola A, Mantero S. Derivation of respiratory signals from multi-lead ECGs. In: Computers in Cardiology 1985. Washington DC: IEEE Compter Society Press, 1985;12:113-16.

[4] Penzel T, McNames J, de Chazal P, Raymond B, Murray A, Moody G. Systematic comparison of different algorithms for apnoea detection based on electrocardiogram recordings. Med. Biol. Eng. Comput. 2002;40:402-7.

[5] Goldberger AL, Amaral LAN, Glass L, Hausdorff JM, Ivanov PC, Mark RG, Mietus JE, Moody GB, Peng CK, Stanley HE. PhysioBank, PhysioToolkit, and PhysioNet: Components of a new research resource for complex physiologic signals. Circulation 2000;101(23):e215-e220 [Circulation Electronic Pages; http://circ.ahajournals.org/ cgi/content/full /101/23/ e215].

[6] Lippman N, Stein KM, Lerman B. Comparison of methods for removal of ectopy in measurement of heart rate variability. Am. J. Physiol. 1994:267;H411-H418.

[7] Garcia J, Lander P, Sörnmo L, Olmos S, Wagner G, Laguna P. Comparative study of local and KarhunenLoève-based ST-T indexes in recordings from subjects with induced myocardial ischemia. Comput. Biomed. Res. 1998;31:271-92.

[8] Maier C, Bauch M, Dickhaus H. Recognition and Quantification of Sleep Apnea by Analysis of Heart Rate Variability Parameters. In: Computers in Cardiology 2000. IEEE Press, 2000;27:741-4.

Address for correspondence.

Christoph Maier

University of Applied Sciences Heilbronn

Max-Planck-Str. 39

D-74081 Heilbronn, Germany

christoph.maier@fh-heilbronn.de 